\title{
Refractory HIV-Associated Lymphoproliferative Disorder
}

National Cancer Institute

\section{Source}

National Cancer Institute. Refractory HIV-Associated Lymphoproliferative Disorder. NCI

Thesaurus. Code C157687.

HIV-associated lymphoproliferative disorder that is resistant to treatment. 